\title{
Global converegence properties of a consensus protocol on the $n$-sphere
}

\author{
Johan Markdahl $^{1}$ and Jorge Goncalves ${ }^{1}$
}

\begin{abstract}
This paper provides a novel analysis of the global convergence properties of a well-known consensus protocol for multi-agent systems that evolve in continuous time on the $n$ sphere. The feedback is intrinsic to the $n$-sphere, i.e., it does not rely on the use of local coordinates obtained through a parametrization. It is shown that, for any connected undirected graph topology and all $n \in \mathbb{N} \backslash\{1\}$, the consensus protocol yields convergence that is akin to almost global consensus in a weak sense. Simulation results suggest that actual almost global consensus holds. This result is of interest in the context of consensus on Riemannian manifolds since it differs from what is known with regard to the 1 -sphere and $\mathrm{SO}(3)$ where more advanced intrinsic consensus protocols are required in order to generate equivalent results.
\end{abstract}

\section{INTRODUCTION}

Consider a network of $N$ agents whose states are points on an $n$-dimensional sphere. Each agent has some limited capability to communicate with and sense relative information of a subset of the other agents. Distributed control protocols allow the agents to synchronize, i.e., to reach a consensus or to propagate information by means of local interactions [1]. This paper establishes some unexpected global stability properties of a well-known consensus protocol. Consensus on the sphere is of interest in a number of applications, including cooperative reduced rigid-body attitude control [2], [3], planetary scale mobile sensing networks [4], and self synchronizing chemical and biological oscillators described by the so-called Kuramoto model [5], [6].

The reduced attitude is a property of objects that for various reasons, such as task redundancy, cylindrical symmetry, or actuator failure, lack one degree of rotational freedom in three-dimensional space and whose orientation corresponds to a pointing direction with no regard for the rotation about the axis of pointing [7]. The reduced attitude synchronization problem is equivalent to the consensus problem on the sphere. The problem of cooperative control on the $n$-sphere, $\mathcal{S}^{n}$, has received some attention in the literature [8]-[10], but comparatively less than equivalent problems on $\mathrm{SO}(3)$ for which there is vast literature including [11]-[21].

The problem of almost global consensus has been studied on $\mathcal{S}^{1}$ [9], on $\mathrm{SO}(3)$ [22], and on $\mathcal{S}^{n}$ in the special case of a complete graph [8], [10]. Tron et al. [22] applies an optimization based method to characterize the stability of all equilibria for a particular discrete-time consensus protocol. Their result is akin to almost global consensus over any connected graph topology. The algorithm uses a particular reshaping function with two parameters, one of which

\footnotetext{
${ }^{1}$ Luxembourg Centre for Systems Biomedicine (LCSB), University of Luxembourg, Belval, Luxembourg.

Email: johan.markdahl@uni.lu, jorge.goncalves@uni.lu
}

must be bounded below. The bound depends on the graph topology, which is unknown to the agents. Moreover, the overall convergence speed of the algorithm decreases with increasing values of the parameter. By shifting consideration from $\mathrm{SO}(3)$ to the $n$-sphere, this paper uses the direct method of Lyapunov to establish stability results of the same type as those in [22] for a well-known consensus protocol that does not require the use of any reshaping function.

Tron et al. [22] divide the literature on discrete-time attitude consensus into two categories: extrinsic and intrinsic algorithms. An algorithm is said to be extrinsic if it relies on the embedding of $\mathrm{SO}(3)$ in an ambient space and then projects its iterates on $\mathrm{SO}(3)$. There are algorithms in this class that provide consensus on a global level. Global level intrinsic consensus on $\mathrm{SO}(3)$ had not been obtained prior to [22]. Much of previous work concerning continuous-time cooperative control on the $n$-sphere regards only the case of a complete graph [8], [10]. Some preliminary results regarding a class of of non-trivial topologies (essentially the case of cycle graphs) is found in the main authors' previous work [2], [3], [23]. The gossip algorithm [9] achieves consensus on $\mathcal{S}^{n}$ with probability 1 for a class of digraphs, but it does not apply to mechanical systems.

The 2-sphere is akin to a subset of $\mathrm{SO}(3)$ and, as such, many results obtained for $\mathrm{SO}(3)$ also apply to $\mathcal{S}^{2}$. Special cases sometimes allow for stronger results. The findings of this paper indicates that the conditions for achieving almost global consensus are, in a certain sense, more favorable on the $n$-sphere for $n \in \mathbb{N} \backslash\{1\}$ than implied by the particularization of previously known results concerning the 1-sphere and $\mathrm{SO}(3)$. A well-known intrinsic consensus protocol over connected, undirected graph topologies renders all equilibria except the consensus point unstable on $\mathcal{S}^{2}$. By contrast, analysis on $\mathcal{S}^{1} \simeq \mathrm{SO}(2)$ [9] and simulation results on $\mathrm{SO}(3)$ [22] show that certain graph topologies yield equilibria that are asymptotically stable on $\mathrm{SO}(n), n \in\{2,3\}$.

The main contribution of this paper is to provide a convergence results on a global level for all connected, undirected graph topologies, i.e., for a much larger class of topologies than what has previously been found on the $n$-sphere [2], [8], [10], [17], [23]. Unlike [22], the control law, which is well-known, does not require the use of a reshaping function depending on the graph topology, i.e., on unavailable information. This result is conjectured in [2], [8], but may be considered unexpected since it is qualitatively different from what is known to hold with regard to discretetime consensus on $\mathcal{S}^{1}$ and on the Lie group $\mathrm{SO}(3)$ of which $\mathcal{S}^{2}$ may be considered a subset [9], [22]. 


\section{PROBLEM DESCRIPTION}

The following notation is used in this paper. The inner product and outer product of $\mathbf{x}, \mathbf{y} \in \mathbb{R}^{n}$ are denoted by $\langle\mathbf{x}, \mathbf{y}\rangle$ and $\mathbf{x} \otimes \mathbf{y}$ respectively. The Euclidean norm is used for vectors and the Frobenius norm is used for matrices. The special orthogonal group is $\mathrm{SO}(n)=\left\{\mathbf{R} \in \mathbb{R}^{n \times n} \mid \mathbf{R}^{-1}=\right.$ $\mathbf{R}^{\top}$, $\left.\operatorname{det} \mathbf{R}=1\right\}$. The Lie algebra of $\mathrm{SO}(n)$ is $\operatorname{so}(n)=$ $\left\{\mathbf{S} \in \mathbb{R}^{n \times n} \mid \mathbf{S}^{\dagger}=-\mathbf{S}\right\}$. The $n$-sphere is denoted by $\mathcal{S}^{n}=\left\{\mathbf{x} \in \mathbb{R}^{n+1} \mid\|\mathbf{x}\|=1\right\}$, where $n \in \mathbb{N}$. We do not consider the trivial 0 -sphere $\mathcal{S}^{0}=\{-1,1\}$. A graph is a pair $\mathcal{G}=(\mathcal{V}, \mathcal{E})$ where $\mathcal{V}$ is the node set and $\mathcal{E} \subset \mathcal{V} \times \mathcal{V}$ is the edge set.

Definition 1. An equilibrium manifold is said to be maximal if it is connected and not a strict subset of any other connected equilibrium manifold.

Definition 2. A maximal equilibrium manifold is said to be uniquely stable if it is stable and no other maximal equilibrium manifold is stable, uniquely attractive if it is attractive and no other maximal equilibrium manifold is attractive, and uniquely asymptotically stable if it is both uniquely stable and uniquely attractive.

\section{A. Distributed Control Design on the n-Sphere}

Consider a multi-agent system where each agent corresponds to an index $i \in \mathcal{V}$. The agents are capable of limited pairwise communication and local sensing. The topology of the communication network is described by an undirected connected graph $\mathcal{G}=(\mathcal{V}, \mathcal{E})$, where $\mathcal{V}=\{1,2, \ldots, N\}$, and $(i, j) \in \mathcal{E}$ implies that agent $i$ and $j$ can communicate, or equivalently that agent $i$ and $j$ can sense the so-called local or relative information, i.e., the displacement of their states.

Control is assumed to be based on relative information and to be carried out on a kinematic level. The information $\mathcal{I}_{i j}$ that agent $i$ has access to regarding its neighbor agent $j$ includes

$$
\operatorname{span}\left\{\left(\mathbf{I}-\mathbf{X}_{i}\right)\left(\mathbf{x}_{j}-\mathbf{x}_{i}\right)\right\}=\operatorname{span}\left\{\mathbf{x}_{j}-\left\langle\mathbf{x}_{j}, \mathbf{x}_{i}\right\rangle \mathbf{x}_{i}\right\},
$$

where $\mathbf{X}_{i}=\mathbf{x}_{i} \otimes \mathbf{x}_{i}$ and $\mathbf{I}-\mathbf{X}_{i}: \mathbb{R}^{n+1} \rightarrow \mathrm{T}_{\mathbf{x}_{i}} \mathcal{S}^{n}$ is a projection. The set of neighbors of agent $i$ is denoted $\mathcal{N}_{i}=$ $\{j \in \mathcal{V} \mid(i, j) \in \mathcal{E}\}$. The space of relative information known to any agent $i \in \mathcal{V}$ is $\cup_{j \in \mathcal{N}_{i}} \mathcal{I}_{i j}$. The subset of $\mathcal{I}_{i j}$ given by (1) corresponds to the customary relative information in linear spaces $\mathcal{I}_{i j} \supset \operatorname{span}\left\{\mathbf{x}_{j}-\mathbf{x}_{i}\right\}$ projected on $\mathrm{T}_{\mathbf{x}_{i}} \mathcal{S}^{n}$. An agent can calculate this aspect of $\mathcal{I}_{i j}$ based on local sensing.

System 3. The system is given by $N$ agents, an undirected and connected graph $\mathcal{G}=(\mathcal{V}, \mathcal{E})$, agent states $\mathbf{x}_{i} \in \mathcal{S}^{n}$, where $n \in \mathbb{N} \backslash\{1\}$, and dynamics

$$
\dot{\mathbf{x}}_{i}=\mathbf{u}_{i}-\left\langle\mathbf{u}_{i}, \mathbf{x}_{i}\right\rangle \mathbf{x}_{i}=\left(\mathbf{I}-\mathbf{X}_{i}\right) \mathbf{u}_{i},
$$

where $\mathbf{u}_{i}: \prod_{j \in \mathcal{N}_{i}} \mathcal{I}_{i j} \rightarrow \mathbb{R}^{n+1}$ is the input signal of agent $i$ and $\mathbf{X}_{i}=\mathbf{x}_{i} \otimes \mathbf{x}_{i}$ for all $i \in \mathcal{V}$.

Note that the dynamics (2) projects the input $\mathbf{u}_{i}$ on the space of relative information. While some agent $i \in \mathcal{V}$ may not be able to calculate some $\mathbf{u}_{i} \in \mathcal{I}_{i j}$ based on the information (1) obtained from all its neighbors, that agent may still be able to calculate an input $\mathbf{v}_{i}$ whose projection on $\mathrm{T}_{\mathbf{x}_{i}} \mathcal{S}^{n}$ is identical to that of $\mathbf{u}_{i}$. This holds for inputs that belongs to $\operatorname{span}\left\{\mathbf{x}_{j} \mid j \in \mathcal{N}_{i}\right\}$, and in particular for elements of the positive cone $\operatorname{pos}\left\{\mathbf{x}_{j} \mid j \in \mathcal{N}_{i}\right\}$. Intuitively speaking, it is reasonable to assume that agent $i$ should be able to move towards any point in $\operatorname{pos}\left\{\mathbf{x}_{j} \mid j \in \mathcal{N}_{i}\right\}$, i.e., that $\operatorname{pos}\left\{\mathbf{x}_{j} \mid j \in \mathcal{N}_{i}\right\} \subset \mathcal{I}_{i j}$.

All vectors in this section are defined in the world frame $\mathcal{W}$. To implement the control law in a distributed fashion, $\mathbf{u}_{i}$ must be transfered to the body frame $\mathcal{B}_{i}$ of agent $i$. Let $[\mathbf{x}]_{\mathcal{F}}$ denote that the coordinates of vector $\mathbf{x}$ are given with respect to the $\mathcal{F}$. Suppose $\mathcal{B}_{i}$ is related to $\mathcal{W}$ by means of a rotation $\mathbf{R}_{i}:[\mathbf{v}]_{\mathcal{W}} \mapsto[\mathbf{v}]_{\mathcal{B}_{i}}$. The control law in $\mathcal{W}$ is given by $\left[\mathbf{u}_{i}\right]_{\mathcal{W}}=\sum_{j \in \mathcal{N}_{i}} w_{i j}\left[\mathbf{x}_{j}\right]_{\mathcal{W}}$. Hence $\left[\mathbf{u}_{i}\right]_{\mathcal{B}_{i}}=$ $\sum_{j \in \mathcal{N}_{i}} w_{i j} \mathbf{R}_{i}\left[\mathbf{x}_{j}\right]_{\mathcal{W}}=\sum_{j \in \mathcal{N}_{i}} w_{i j}\left[\mathbf{x}_{j}\right]_{\mathcal{B}_{i}}$. Moreover,

$$
\begin{aligned}
{\left[\dot{\mathbf{x}}_{i}\right]_{\mathcal{B}_{i}} } & =\mathbf{R}_{i}\left[\mathbf{u}_{i}\right]_{\mathcal{W}}-\left\langle\left[\mathbf{u}_{i}\right]_{\mathcal{W}}, \mathbf{R}_{i}^{\top} \mathbf{R}_{i}\left[\mathbf{x}_{i}\right]_{\mathcal{W}}\right\rangle \mathbf{R}_{i}\left[\mathbf{x}_{i}\right]_{\mathcal{W}} \\
& =\left[\mathbf{u}_{i}\right]_{\mathcal{B}_{i}}-\left\langle\left[\mathbf{u}_{i}\right]_{\mathcal{B}_{i}},\left[\mathbf{x}_{i}\right]_{\mathcal{B}_{i}}\right\rangle\left[\mathbf{x}_{i}\right]_{\mathcal{B}_{i}}
\end{aligned}
$$

due to inner products being invariant under orthonormal changes of coordinates.

The problem of multi-agent consensus on the $n$-sphere concerns the design of distributed control protocols $\left\{\mathbf{u}_{i}\right\}_{i=1}^{N}$ based on relative information, as discussed in the above paragraphs, that stabilize the consensus manifold

$$
\mathcal{C}=\left\{\left\{\mathbf{x}_{i}\right\}_{i=1}^{N} \in\left(\mathcal{S}^{n}\right)^{N} \mid \mathbf{x}_{i}=\mathbf{x}_{j}, \forall i, j \in \mathcal{V}\right\}
$$

of System 3. If all agents converge to one point on the $n$-sphere, then they are said to reach consensus. For all connected graphs, it can easily be shown to suffice that the states of any pair of neighboring agents are equal.

\section{B. Problem Statement}

This paper concerns some aspects of control design but the main focus is stability analysis for a linear consensus protocol on the $n$-sphere. Our work is motivated by a desire to establish a more basic protocol for almost global consensus than that which the particularization of [22] to the $n$-sphere would yield. As such, the use a linear, timeinvariant feedback is very appealing.

Problem 4. Show that there is a linear intrinsic consensus protocol, i.e., linear input signals $\mathbf{u}_{i}: \prod_{j \in \mathcal{N}_{i}} \mathcal{I}_{i j} \rightarrow \mathbb{R}^{n+1}$ for all $i \in \mathcal{V}$, for System 3 such that the consensus manifold is uniquely asymptotically stable.

Problem 4 concerns the global behavior of the system. Under certain assumptions regarding the connectivity of $\mathcal{G}$, local consensus on $\mathrm{SO}(3)$ can be established with the region of attraction being the largest geodesically convex sets on $\mathcal{S}^{n}$, i.e., open hemispheres. See for example [17] in the case of an undirected graph and [20] in the case of a directed and time-varying graph. A global stability result for discretetime consensus on $\mathrm{SO}(3)$ is provided in [22]. Almost global asymptotical stability of the consensus manifold on the $n$ sphere is known to hold when the graph is a tree [17] or is complete in the case of first- and second-order models [8], 
[10]. The author of [8] conjectures that global stability also holds for a larger class of topologies whereas [9] provides counter-examples of basic consensus protocols that fail to generate consensus on $\mathcal{S}^{1}$.

Remark 5. Global consensus on $\mathcal{S}^{n}$ cannot be achieved by means of a continuous feedback due to topological constraints [27]. It is however possible to achieve almost global asymptotical stability [9]. Tron et al. [22] argue that adding a small perturbation to the feedback of a uniquely asymptotically stable system yields almost global consensus. However, that is not true in general. To prove almost global consensus is difficult since basic tools such as the HartmanGrobman theorem or stable-unstable manifold theorems are unavailable due to the equilibria being nonhyperbolic [28]. Instead, center manifold theory for equilibrium manifolds may be required. That is the topic of future work, whereas this paper deals with unique asymptotical stability.

\section{Stability of Desired Equilibria}

This section concerns System 3 governed by Algorithm 6 below which provides a smooth protocol for continuous-time consensus on the $n$-sphere. It can be argued that Algorithm 6 is the most basic conceivable feedback for this problem by virtue of its correspondence with the linear consensus protocol given by $\mathbf{u}_{i}=\sum_{i \in \mathcal{N}_{i}} w_{i j}\left(\mathbf{x}_{j}-\mathbf{x}_{i}\right)$ where $w_{i j} \in$ $(0, \infty), w_{i j}=w_{j i}$ for all $(i, j) \in \mathcal{E}$. This follows since the dynamics on the $n$-sphere project an input to the orthogonal complement of the state of each agent.

\section{A. Previous Research}

Algorithm 6. The feedback is given by $\mathbf{u}_{i}=\sum_{j \in \mathcal{N}_{i}} w_{i j} \mathbf{x}_{j}$, where $w_{i j} \in(0, \infty)$ and $w_{i j}=w_{j i}$ for all $(i, j) \in \mathcal{E}$.

Algorithm 6 is well-known and can be derived by taking the gradient of the potential function

$$
V=\frac{1}{2} \sum_{(i, j) \in \mathcal{E}} w_{i j}\left\|\mathbf{x}_{i}-\mathbf{x}_{j}\right\|^{2}=\sum_{(i, j) \in \mathcal{E}} w_{i j}\left(1-\cos \vartheta_{i j}\right),
$$

where $\vartheta_{i j}$ is the angle between $\mathbf{x}_{i}, \mathbf{x}_{j} \in \mathcal{S}^{n}$. It is possible to work with more general gains than the constant weights of Algorithm 6, but we prefer the use of a linear feedback. Proposition 7 implies that there are no limits cycles in System 3 under Algorithm 6. If the graph in System 3 under Algorithm 6 were directed, then there would exist examples of topologies that result in limit cycles.

Proposition 7. System 3 converges to an equilibrium manifold. The following equilibrium configurations exist:

$$
\left(\mathbf{x}_{i}, \mathbf{u}_{i}\right) \in\left\{\left(-\frac{\mathbf{u}_{i}}{\left\|\mathbf{u}_{i}\right\|}, \mathbf{u}_{i}\right),\left(\frac{\mathbf{u}_{i}}{\left\|\mathbf{u}_{i}\right\|}, \mathbf{u}_{i}\right),\left(\mathbf{x}_{i}, \mathbf{0}\right)\right\},
$$

where $\mathbf{u}_{i}=\sum_{j \in \mathcal{N}_{i}} w_{i j} \mathbf{x}_{j}$ for all $i \in \mathcal{V}$.

Proof. Consider the potential function (5). It holds that

$$
\begin{aligned}
\dot{V} & =\sum_{(i, j) \in \mathcal{E}} w_{i j}\left\langle\mathbf{x}_{i}-\mathbf{x}_{j}, \dot{\mathbf{x}}_{i}-\dot{\mathbf{x}}_{j}\right\rangle \\
& =\sum_{(i, j) \in \mathcal{E}} w_{i j}\left\langle\mathbf{x}_{i}-\mathbf{x}_{j},\left(\mathbf{I}-\mathbf{X}_{i}\right) \mathbf{u}_{i}-\left(\mathbf{I}-\mathbf{X}_{j}\right) \mathbf{u}_{j}\right\rangle
\end{aligned}
$$

$$
\begin{aligned}
& =-\sum_{(i, j) \in \mathcal{E}} w_{i j}\left[\left\langle\mathbf{x}_{i},\left(\mathbf{I}-\mathbf{X}_{j}\right) \mathbf{u}_{j}\right\rangle+\left\langle\mathbf{x}_{j},\left(\mathbf{I}-\mathbf{X}_{i}\right) \mathbf{u}_{i}\right\rangle\right] \\
& =-\sum_{i \in V} \sum_{j \in \mathcal{N}_{i}} w_{i j}\left\langle\mathbf{x}_{j},\left(\mathbf{I}-\mathbf{X}_{i}\right) \mathbf{u}_{i}\right\rangle \\
& =-\sum_{i \in V}\left\langle\sum_{j \in \mathcal{N}_{i}} w_{i j} \mathbf{x}_{j},\left(\mathbf{I}-\mathbf{X}_{i}\right) \mathbf{u}_{i}\right\rangle \\
& =-\sum_{i \in V}\left\langle\mathbf{u}_{i},\left(\mathbf{I}-\mathbf{X}_{i}\right) \mathbf{u}_{i}\right\rangle=-\sum_{i \in V}\left\|\mathbf{u}_{i}\right\|^{2}-\left\langle\mathbf{u}_{i}, \mathbf{x}_{i}\right\rangle^{2} .
\end{aligned}
$$

System 3 converges to the set $\left\{\left\{\mathbf{x}_{i}\right\}_{i=1}^{N} \mid \mathbf{u}_{i} \| \mathbf{x}_{i}\right\}$ by LaSalle's theorem, i.e., the input and state of each agent align up to sign. This implies $\dot{\mathbf{x}}_{i}=\mathbf{0}$ for all $i \in \mathcal{V}$, i.e., that the system is at an equilibrium by inspection of (2).

The following result, Theorem 8 , may be considered as one of the many known facts concerning consensus on convex subsets of manifolds [17]. To solve Problem 4, this paper provides a companion to Theorem 8 , Theorem 9 below, that regards all equilibrium manifolds of System 3 under Algorithm 6.

Theorem 8. Consider System 3 under Algorithm 6. The system reaches consensus asymptotically if and only if there is some finite time such that all agents belong to an open hemisphere.

\section{B. Main Result}

Theorem 9. The consensus manifold $\mathcal{C}$ of System 3 on $\mathcal{S}^{n}$, where $n \in \mathbb{N} \backslash\{1\}$, is uniquely asymptotically stable under Algorithm 6.

The proof of Theorem 9 is given at the end of Section IV. Let us briefly sketch the main ideas. The requirements of Definition 2 must be satisfied. That the consensus manifold is asymptotically stable follows from the known result of Theorem 8. To prove the instability of the undesired equilibria we use the indirect method of Lyapunov. The system is linearized around an equilibrium on the $n$-sphere. The three types of equilibria given by Proposition 7 are analyzed separately. The cases of $\mathbf{x}_{i}=-\mathbf{u}_{i} /\left\|\mathbf{u}_{i}\right\|$ and $\mathbf{u}_{i}=\mathbf{0}$ for some $i \in \mathcal{V}$ result in matrices with zero or positive diagonal elements that are at least indefinite. Neighbors tend to be close in the case of $\mathbf{x}_{i}=\mathbf{u}_{i} /\left\|\mathbf{u}_{i}\right\|$ for all $i \in \mathcal{V}$. Perturbing all agents towards the north pole increases cohesion in the north hemisphere while depleting it in the south. One such perturbation corresponds to a direction of instability for the linearized system. The instability of equilibria is inherited by the equilibrium manifolds they constitute due to the properties of gradient systems.

\section{InSTABILITy of UndesiRed EQUILIBRIA}

The global behavior of the system is determined by the stability of the equilibrium configurations given in Proposition 7. Set all weights in Algorithm 6 to be equal. An example of $\mathbf{x}_{i}=-\mathbf{u}_{i} /\left\|\mathbf{u}_{i}\right\|$ is given by a tetrahedron formation with a tetrahedral graph, i.e., the complete graph on four nodes. An example of $\mathbf{x}_{i}=\mathbf{0}$ for all $i \in \mathcal{V}$ is provided by the octahedral graph, see Figure 1. The case of $\mathbf{x}_{i}=\mathbf{u}_{i} /\left\|\mathbf{u}_{i}\right\|$ for 
all $i \in \mathcal{V}$ poses a more difficult challenge since it includes the consensus manifold as a special case. The difficulty is overcome by the indirect method of Lyapunov. As such, we study the signs of the real part of the linearization of System 3 under Algorithm 6 in order to establish the instability of all undesired equilibria and equilibrium manifolds.
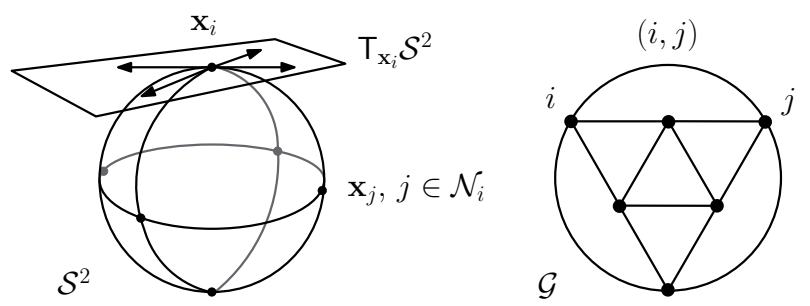

Fig. 1. An unstable equilibrium of a system on $\mathcal{S}^{2}$ (left) with an octahedral graph (right). The sum of neighbor states projected on the tangent plane $\mathrm{T}_{\mathbf{x}_{i}} \mathcal{S}^{2}$ is zero (left).

Proposition 10. The $(n+1) \times(n+1)$ blocks of the $N(n+$ $1) \times N(n+1)$ matrix $\mathbf{A}$ that describes the linearization on $\mathcal{S}^{n}$ of System 3 under Algorithm 6 are given by

$$
\mathbf{A}_{i j}= \begin{cases}-\left(\left\langle\mathbf{u}_{i}, \mathbf{x}_{i}\right\rangle \mathbf{I}+\mathbf{x}_{i} \otimes \mathbf{u}_{i}\right)\left(\mathbf{I}-\mathbf{X}_{i}\right) & \text { if } j=i, \\ w_{i j}\left(\mathbf{I}-\mathbf{X}_{i}\right)\left(\mathbf{I}-\mathbf{X}_{j}\right) & \text { if } j \neq i,\end{cases}
$$

for $(i, j) \in \mathcal{E}$ and $\mathbf{A}_{i j}=\mathbf{0}$ otherwise. The matrix $\mathbf{A}$ is symmetric.

Proof. For systems evolving on manifolds, a perturbation technique is used to obtain the linearized dynamics. Let $\mathbf{x}_{i}$ for all $i \in \mathcal{V}$ be a solution to (2). Consider a perturbed solution $\mathbf{x}_{i}\left(\varepsilon, \mathbf{v}_{i}\right)$ given by

$$
\mathbf{x}_{i}\left(\varepsilon, \mathbf{v}_{i}\right)=\frac{\mathbf{x}_{i}+\varepsilon \mathbf{v}_{i}}{\left\|\mathbf{x}_{i}+\varepsilon \mathbf{v}_{i}\right\|},
$$

where $\mathbf{v}_{i}$ is a smooth function. The perturbed solution is required to satisfy the differential equation

$$
\dot{\mathbf{x}}_{i}\left(\varepsilon, \mathbf{v}_{i}\right)=\mathbf{u}_{i}\left(\varepsilon, \mathbf{v}_{i}\right)-\left\langle\mathbf{u}_{i}\left(\varepsilon, \mathbf{v}_{i}\right), \mathbf{x}_{i}\left(\varepsilon, \mathbf{v}_{i}\right)\right\rangle \mathbf{x}_{i}\left(\varepsilon, \mathbf{v}_{i}\right) .
$$

The linearized dynamics on $\mathcal{S}^{n}$ can be derived by studying the linear effect of $\mathbf{v}_{i}$ on $\dot{\mathbf{x}}_{i}\left(\varepsilon, \mathbf{v}_{i}\right)$. Define

$$
\begin{aligned}
\mathbf{w}_{i}= & \left.\frac{\mathrm{d}}{\mathrm{d} \varepsilon} \mathbf{x}_{i}\left(\varepsilon, \mathbf{v}_{i}\right)\right|_{\varepsilon=0}=\left.\frac{\mathbf{v}_{i}}{\left\|\mathbf{x}_{i}+\varepsilon \mathbf{v}_{i}\right\|}\right|_{\varepsilon=0}- \\
& \left.\frac{\mathbf{x}_{i}+\varepsilon \mathbf{v}_{i}}{\left\|\mathbf{x}_{i}+\varepsilon \mathbf{v}_{i}\right\|^{3}}\left\langle\mathbf{x}_{i}, \mathbf{v}_{i}\right\rangle\right|_{\varepsilon=0} \\
= & \mathbf{v}_{i}-\mathbf{x}_{i} \otimes \mathbf{x}_{i} \mathbf{v}_{i}=\left(\mathbf{I}-\mathbf{X}_{i}\right) \mathbf{v}_{i},
\end{aligned}
$$

where $\mathbf{X}_{i}=\mathbf{x}_{i} \otimes \mathbf{x}_{i}$. The matrix $\mathbf{I}-\mathbf{X}_{i}$ projects onto the tangent space $\mathrm{T}_{\mathbf{x}_{i}} \mathcal{S}^{n}$ where $\mathbf{w}_{i}$ lives. Note that

$$
\left.\frac{\mathrm{d}}{\mathrm{d} \varepsilon} \mathbf{X}_{i}\left(\varepsilon, \mathbf{v}_{i}\right)\right|_{\varepsilon=0}=\mathbf{w}_{i} \otimes \mathbf{x}_{i}+\mathbf{x}_{i} \otimes \mathbf{w}_{i}
$$

by the product rule. Then

$$
\begin{aligned}
\dot{\mathbf{w}}_{i} & =\left.\frac{\mathrm{d}^{2}}{\mathrm{~d} t \mathrm{~d} \varepsilon} \mathbf{x}_{i}\left(\varepsilon, \mathbf{v}_{i}\right)\right|_{\varepsilon=0}=\left.\frac{\mathrm{d}}{\mathrm{d} \varepsilon} \dot{\mathbf{x}}_{i}\left(\varepsilon, \mathbf{v}_{i}\right)\right|_{\varepsilon=0} \\
& =\frac{\mathrm{d}}{\mathrm{d} \varepsilon}\left(\mathbf{I}-\mathbf{X}_{i}\left(\varepsilon, \mathbf{v}_{i}\right)\right) \sum_{j \in \mathcal{N}_{i}} w_{i j} \mathbf{x}_{j}\left(\varepsilon, \mathbf{v}_{j}\right)
\end{aligned}
$$

$$
\begin{aligned}
= & -\left.\left[\frac{\mathrm{d}}{\mathrm{d} \varepsilon} \mathbf{X}_{i}\left(\varepsilon, \mathbf{v}_{i}\right)\right] \sum_{j \in \mathcal{N}_{i}} w_{i j} \mathbf{x}_{j}\left(\varepsilon, \mathbf{v}_{j}\right)\right|_{\varepsilon=0}+ \\
& \left.\left(\mathbf{I}-\mathbf{X}_{i}\left(\varepsilon, \mathbf{v}_{i}\right)\right) \sum_{j \in \mathcal{N}_{i}} w_{i j} \frac{\mathrm{d}}{\mathrm{d} \varepsilon} \mathbf{x}_{j}\left(\varepsilon, \mathbf{v}_{j}\right)\right|_{\varepsilon=0} \\
= & -\left(\mathbf{w}_{i} \otimes \mathbf{x}_{i}+\mathbf{x}_{i} \otimes \mathbf{w}_{i}\right) \sum_{j \in \mathcal{N}_{i}} \mathbf{x}_{j}+ \\
& \left(\mathbf{I}-\mathbf{X}_{i}\right) \sum_{j \in \mathcal{N}_{i}} w_{i j} \mathbf{w}_{j} \\
= & -\left(\mathbf{w}_{i} \otimes \mathbf{x}_{i}+\mathbf{x}_{i} \otimes \mathbf{w}_{i}\right) \mathbf{u}_{i}+\left(\mathbf{I}-\mathbf{X}_{i}\right) \sum_{j \in \mathcal{N}_{i}} w_{i j} \mathbf{w}_{j} \\
= & -\left(\left\langle\mathbf{u}_{i}, \mathbf{x}_{i}\right\rangle \mathbf{I}+\mathbf{x}_{i} \otimes \mathbf{u}_{i}\right) \mathbf{w}_{i}+ \\
& \left(\mathbf{I}-\mathbf{X}_{i}\right) \sum_{j \in \mathcal{N}_{i}} w_{i j} \mathbf{w}_{j} \\
= & -\left(\left\langle\mathbf{u}_{i}, \mathbf{x}_{i}\right\rangle \mathbf{I}+\mathbf{x}_{i} \otimes \mathbf{u}_{i}\right)\left(\mathbf{I}-\mathbf{X}_{i}\right) \mathbf{v}_{i}+ \\
& \left(\mathbf{I}-\mathbf{X}_{i}\right) \sum_{j \in \mathcal{N}_{i}} w_{i j}\left(\mathbf{I}-\mathbf{X}_{j}\right) \mathbf{v}_{j}
\end{aligned}
$$

where the relation $\langle\mathbf{x}, \mathbf{y}\rangle \mathbf{z}=(\mathbf{z} \otimes \mathbf{x}) \mathbf{y}=(\mathbf{z} \otimes \mathbf{y}) \mathbf{x}$ for all $\mathbf{x}, \mathbf{y}, \mathbf{z} \in \mathbb{R}^{n+1}$ is used. The vector $\mathbf{w}=\left[\mathbf{w}_{1}^{\top} \ldots \mathbf{w}_{N}^{\top}\right]^{\top}$ has $N(n+1)$ components whereas the linearized system actually evolves on an $N n$-dimensional space that lies embedded in $\mathbb{R}^{N(n+1)}$. The dimension reduction is given implicitly by the definition of $\mathbf{w}_{i}$ which requires $\mathbf{w}_{i} \in \mathrm{T}_{\mathbf{x}_{i}} \mathcal{S}^{n}$. This constraint is removed by using variables that are premultiplied by the projection matrices $\mathbf{I}-\mathbf{X}_{i}: \mathbb{R}^{n+1} \rightarrow \mathrm{T}_{\mathbf{x}_{i}} \mathcal{S}^{n}$, i.e., the variables $\mathbf{v}_{i}$ in (6).

It remains to show that $\mathbf{A}$ is symmetric. Write

$$
\begin{aligned}
\mathbf{A}_{i i} & =-\left(\mathbf{x}_{i} \otimes \mathbf{u}_{i}+\left\langle\mathbf{u}_{i}, \mathbf{x}_{i}\right\rangle \mathbf{I}\right)\left(\mathbf{I}-\mathbf{X}_{i}\right) \\
& =-\left(\mathbf{x}_{i} \otimes \mathbf{u}_{i}+\mathbf{u}_{i} \otimes \mathbf{x}_{i}+\left\langle\mathbf{u}_{i}, \mathbf{x}_{i}\right\rangle \mathbf{I}\right)\left(\mathbf{I}-\mathbf{X}_{i}\right)
\end{aligned}
$$

which is clearly symmetric. Moreover,

$$
\begin{aligned}
\mathbf{A}_{j i}^{\top}-\mathbf{A}_{i j}= & w_{j i}\left[\left(\mathbf{I}-\mathbf{X}_{j}\right)\left(\mathbf{I}-\mathbf{X}_{i}\right)\right]^{\top}- \\
& w_{i j}\left(\mathbf{I}-\mathbf{X}_{i}\right)\left(\mathbf{I}-\mathbf{X}_{j}\right)=\mathbf{0},
\end{aligned}
$$

since $w_{i j}=w_{j i}$ for all $(i, j) \in \mathcal{E}$.

\section{A. Unstable Equilibria}

Proposition 11. Any equilibrium $\left\{\mathbf{x}_{i}\right\}_{i=1}^{N} \notin \mathcal{C}$ of System 3 where $n \in \mathbb{N} \backslash\{1\}$ under Algorithm 6 is unstable.

Proof. The proof makes use of the linearization provided by Proposition 10. The Courant-Fischer-Weyl min-max principle bounds the range of the Rayleigh quotient of a symmetric matrix by its minimal and maximal eigenvalues [29]. If the Rayleigh quotient is positive for some argument, then the maximal eigenvalue is positive. Recall that if $\mathbf{A}$ has a positive eigenvalue at an equilibrium, then that equilibrium is unstable by the indirect method of Lyapunov [30].

In the case of $\mathbf{x}_{i}=-\mathbf{u}_{i} /\left\|\mathbf{u}_{i}\right\|$ for some $i \in \mathcal{V}$, note that the diagonal block of the linearization matrix $\mathbf{A}$ is given by $\mathbf{A}_{i i}=\left\|\mathbf{u}_{i}\right\|\left(\mathbf{I}-\mathbf{X}_{i}\right)$, which is a positive semidefinite, nonzero matrix. There is hence an eigenpair $(\lambda, \mathbf{y})$ of $\mathbf{A}_{i i}$ with $\lambda \in(0, \infty), \mathbf{y} \in \mathcal{S}^{n}$. Let $\mathbf{v} \in \mathbb{R}^{N(n+1)}$ denote a vector composed of the blocks $\mathbf{v}_{1}, \ldots, \mathbf{v}_{N} \in \mathbb{R}^{n+1}$. Set $\mathbf{v}_{i}=\mathbf{y}$ 
and $\mathbf{v}_{j}=\mathbf{0}$ for all $j \in \mathcal{V} /\{i\}$ whereby $\langle\mathbf{v}, \mathbf{A} \mathbf{v}\rangle=\lambda$. The largest eigenvalue of $\mathbf{A}$ is hence positive number.

In the case of $\mathbf{u}_{i}=\mathbf{0}$, take a $j \in \mathcal{N}_{i}$ and set $\mathbf{v}_{j}=\varepsilon \mathbf{y}$ for a $\mathbf{y} \in \mathcal{S}^{n}$ such that $\left\langle\mathbf{y}, \mathbf{x}_{i}\right\rangle=0,\left\langle\mathbf{y}, \mathbf{x}_{j}\right\rangle=0$ and an $\varepsilon \in$ $(0, \infty)$. Set $\mathbf{v}_{i}=\mathbf{y}$ and let the other entries of $\mathbf{v}$ be zeros. Then $\langle\mathbf{v}, \mathbf{A} \mathbf{v}\rangle=\left\langle\mathbf{y}, \mathbf{A}_{j j} \mathbf{y}\right\rangle \varepsilon^{2}+2 w_{i j} \varepsilon$, which is positive given that $\varepsilon$ is sufficiently small.

It remains to consider the case of $\mathbf{x}_{i}=\mathbf{u}_{i} /\left\|\mathbf{u}_{i}\right\|$. Then $\mathbf{u}_{i}=\left\|\mathbf{u}_{i}\right\| \mathbf{x}_{i}$ and hence

$$
\mathbf{A}_{i j}= \begin{cases}-\left\|\mathbf{u}_{i}\right\|\left(\mathbf{I}-\mathbf{X}_{i}\right) & \text { if } j=i \\ w_{i j}\left(\mathbf{I}-\mathbf{X}_{i}\right)\left(\mathbf{I}-\mathbf{X}_{j}\right) & \text { if } j \neq i\end{cases}
$$

for $(i, j) \in \mathcal{E}$ and $\mathbf{A}_{i j}=\mathbf{0}$ otherwise. The matrix $\mathbf{A}$ is symmetric at all equilibria by Proposition 10 .

Let $\mathbf{v}=\left[\mathbf{y}^{\top} \ldots \mathbf{y}^{\top}\right]^{\top}$ and consider

$$
\begin{aligned}
\langle\mathbf{v}, \mathbf{A} \mathbf{v}\rangle & =\sum_{i \in \mathcal{V}}\left\langle\mathbf{y}, \mathbf{A}_{i i} \mathbf{y}\right\rangle+\sum_{j \in \mathcal{N}_{i}}\left\langle\mathbf{y}, \mathbf{A}_{i j} \mathbf{y}\right\rangle \\
& =\left\langle\mathbf{y},\left(\sum_{i \in \mathcal{V}} \mathbf{A}_{i i}+\sum_{j \in \mathcal{N}_{i}} \mathbf{A}_{i j}\right) \mathbf{y}\right\rangle
\end{aligned}
$$

Denote $\mathbf{B}=\sum_{i \in \mathcal{V}} \mathbf{A}_{i i}+\sum_{j \in \mathcal{N}_{i}} \mathbf{A}_{i j}$. The matrix $\mathbf{B}$ is symmetric due to $\mathbf{A}$ being symmetric whereby $\sigma(\mathbf{B}) \subset \mathbb{R}$ by the spectral theorem. If $(\lambda, \mathbf{z})$ is an eigenpair of $\mathbf{B}$, then $\left(\lambda,\left[\mathbf{z}^{\top} \ldots \mathbf{z}^{\top}\right]^{\top}\right)$ is an eigenpair of $\mathbf{A}$.

Let us prove that $\mathbf{B}$ has a positive eigenvalue, which in turn will imply that $\mathbf{A}$ has a positive eigenvalue. Consider

$$
\begin{aligned}
\operatorname{tr} \mathbf{B}= & -\sum_{i \in \mathcal{V}}\left\|\mathbf{u}_{i}\right\|\left(n+1-\operatorname{tr} \mathbf{X}_{i}\right)+ \\
& \sum_{j \in \mathcal{N}_{i}} w_{i j}\left(n+1-\operatorname{tr} \mathbf{X}_{i}-\operatorname{tr} \mathbf{X}_{j}+\operatorname{tr} \mathbf{X}_{i} \mathbf{X}_{j}\right) \\
= & -\sum_{i \in \mathcal{V}} n\left\|\mathbf{u}_{i}\right\|+\sum_{j \in \mathcal{N}_{i}} w_{i j}\left(n-1+\left\langle\mathbf{x}_{i}, \mathbf{x}_{j}\right\rangle^{2}\right) \\
= & n\left(\sum_{i \in \mathcal{V}}-\left\|\mathbf{u}_{i}\right\|+\sum_{j \in \mathcal{N}_{i}} w_{i j}\right)- \\
& \sum_{i \in \mathcal{V}} \sum_{j \in \mathcal{N}_{i}} w_{i j}\left(1-\cos { }^{2} \vartheta_{i j}\right) \\
= & n\left(\sum_{i \in \mathcal{V}} \sum_{j \in \mathcal{N}_{i}} w_{i j}\left(1-\cos \vartheta_{i j}\right)\right)- \\
& \sum_{i \in \mathcal{V}} \sum_{j \in \mathcal{N}_{i}} w_{i j}\left(1+\cos \vartheta_{i j}\right)\left(1-\cos \vartheta_{i j}\right) \\
= & \sum_{i \in \mathcal{V}} \sum_{j \in \mathcal{N}_{i}} w_{i j}\left(n-1-\cos \theta_{i j}\right)\left(1-\cos \vartheta_{i j}\right),
\end{aligned}
$$

where we use $\operatorname{tr} \mathbf{X}_{i}=\left\|\mathbf{x}_{i}\right\|^{2}=1$ and $\left\|\mathbf{u}_{i}\right\|=\left\langle\mathbf{x}_{i}, \mathbf{u}_{i}\right\rangle=$ $\sum_{j \in \mathcal{N}_{i}}\left\langle\mathbf{x}_{i}, w_{i j} \mathbf{x}_{j}\right\rangle=\sum_{j \in \mathcal{N}_{i}} w_{i j} \cos \vartheta_{i j}$ and denote $\theta_{i j}=$ $\operatorname{acos}\left\langle\mathbf{x}_{i}, \mathbf{x}_{j}\right\rangle$. Since $\operatorname{tr} \mathbf{B} \geq 0$ for all $n \in \mathbb{N} \backslash\{1\}$ with strict inequality unless $\theta_{i j}=0$ for all $(i, j) \in \mathcal{E}$, i.e., unless $\left\{\mathbf{x}_{i}\right\}_{i=1}^{N} \in \mathcal{C}$, it follows that $\mathbf{B}$ has an eigenvalue $\lambda \in(0, \infty)$.

\section{B. Unstable Equilibrium Manifolds}

Proposition 12. Any connected equilibrium manifold $\mathcal{M} \not \subset$ $\mathcal{C}$ of System 3 where $n \in \mathbb{N} \backslash\{1\}$ under Algorithm 6 is unattractive.

Proof. Consider an equilibrium $\left\{\mathbf{x}_{i}\right\}_{i=1}^{N} \in \mathcal{M}$. Since $\left\{\mathbf{x}_{i}\right\}_{i=1}^{N}$ is unstable by Proposition 11 , there must be a smooth trajectory $\mathcal{T}:(0, \infty) \rightarrow\left(\mathcal{S}^{n}\right)^{N}$ with $\mathcal{T}(0) \notin$ $\mathcal{M}$ such that, for some $\varepsilon \in(0, \infty)$, there is no $\delta \in$ $(0, \infty)$ that would make $d\left(\mathcal{T}(0),\left\{\mathbf{x}_{i}\right\}_{i=1}^{N}\right)<\delta$ imply $\sup _{t \in(0, \infty)} d\left(\mathcal{T}(t),\left\{\mathbf{x}_{i}\right\}_{i=1}^{N}\right)<\varepsilon$. Suppose that the trajectory returns to the manifold, i.e., $\lim _{t \rightarrow \infty} \mathcal{T}(t) \in \mathcal{M}$. The potential function $V$ given by (5) decreases along every trajectory of the system but is constant over each connected equilibrium manifold. If the value of $V$ is lower on some point on $\mathcal{T}$ than it is on $\mathcal{M}$, then $\mathcal{T}$ cannot return to $\mathcal{M}$. If $V$ is higher on some point on $\mathcal{T}$ than on $\mathcal{M}$, then it increases even higher backwards in time and by moving $\mathcal{T}(0)$ arbitrarily close to $\mathcal{M}$, the continuity of $V$ is contradicted. Finally, if $V$ is constant on $\mathcal{T}$ and $\lim _{t \rightarrow \infty} \mathcal{T}(t) \in \mathcal{M}$, then $\mathcal{T}(0) \in \mathcal{T} \subset \mathcal{M}$, a contradiction.

Proposition 13. Any maximal equilibrium manifold $\mathcal{M} \not \subset \mathcal{C}$ of System 3 where $n \in \mathbb{N} \backslash\{1\}$ under Algorithm 6 is unstable.

Proof. Reasoning as in the proof of Proposition 12 we find that there is a smooth trajectory $\mathcal{T}:(0, \infty) \rightarrow\left(\mathcal{S}^{n}\right)^{N}$, where $\mathcal{T}(0)$ can be chosen arbitrarily close to an unstable equilibrium $\left\{\mathbf{x}_{i}\right\}_{i=1}^{N} \in \mathcal{M}$, that leaves an $\varepsilon$-neighborhood of $\left\{\mathbf{x}_{i}\right\}_{i=1}^{N}$ for some fixed $\varepsilon \in(0, \infty)$. Suppose that this trajectory stays in an $\varepsilon$-neighborhood of $\mathcal{M}$. It must converge to an equilibrium that does not belong to $\mathcal{M}$ by Proposition 7 and 12. Hence there is a second equilibrium manifold $\mathcal{N}$ at a distance of at most $\varepsilon$ from $\mathcal{M}$. Since $\varepsilon$ is arbitrary, there must be some point where $d(\mathcal{N}, \mathcal{M})=0$. Since $\mathcal{M}$ is closed, this contradicts the assumption of $\mathcal{M}$ being maximal.

We are now ready to prove the main result of this paper, Theorem 9 in Section III-B.

Proof of Theorem 9. The proof follows directly by verifying that the requirements of Definition 2 are satisfied as is evident by Theorem 8 and Proposition 12 and 13 .

\section{Simulation}

The question of whether Theorem 9 can be extended to the case of almost global consensus remains unanswered. This section provides simulations that argue in favor of such a result. Let $\mathcal{R}$ denote the union of the regions of attraction over all equilibrium manifolds except the consensus manifold. Suppose the probability measure of $\mathcal{R}$ on $\left(\mathcal{S}^{2}\right)^{N}$ is $\varepsilon \in[0,1]$. Consider the statement ' $\mathcal{R}$ has measure zero' as a null hypothesis. Let the random variable $X$ denote the number of draws from $\mathcal{R}$ after $m$ draws from $\mathcal{U}\left(\mathcal{S}^{2}\right)$. If $X>0$ then the null hypothesis is rejected. The probability of the null hypothesis not being rejected is $P(X=0)=(1-p)^{m}$, where $p$ is the probability of an outcome belonging to $\mathcal{R}$, i.e., $p=\varepsilon /(4 \pi N)$. If $m$ is large and the null hypothesis is not rejected, then it is very likely that $p$ is small. Of course, the 
expression for $P(X=0)$ does not account for the effect of numerical errors, but convergence to the desired manifold in the presence of errors is anyhow a positive quality.

Good conditions for stable non-consensus equilibria on the 2 -sphere are given by the Platonic solids. The Platonic solids are symmetric three-dimensional bodies whose corners lie on the surface of a sphere. There are a total of five platonic bodies: The tetrahedron, hexahedron, octahedron, icosahedron and dodecahedron (see Figure 1 and 2). A planar depiction of all graphs is given in Table I. If the number of agents equals the number of nodes of a Platonic graph and are positioned on the corners of the corresponding Platonic solid, then the multi-agent system is at an equilibrium since the contributions of all neighboring agents on each tangent space cancel for reasons of symmetry (see Figure 1).
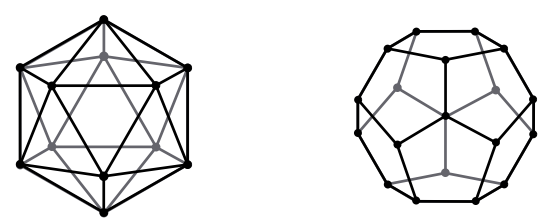

Fig. 2. The icosahedral (left) and dodecahedral (right) graph depicted in three dimensions. All nodes touch the minimal circumscribing sphere.

Table I displays the outcome of running $10^{6}$ trials of Algorithm 6 with $w_{i j}=5$ for all $(i, j) \in \mathcal{E}$ on System 3 for the five platonic graphs. The initial conditions are drawn uniformly from the sphere using the fact that $\mathrm{x} \in \mathcal{N}(\mathbf{0}, \mathbf{I})$ implies that $\mathbf{x} /\|\mathbf{x}\| \in \mathcal{U}\left(\mathcal{S}^{n}\right)$, see [31]. Table I shows that there were no failures to reach consensus. There is hence no reason to reject the null hypothesis. It can be shown, as a consequence of Proposition 11, that the platonic body configurations in the case of the tetrahedron and octahedron are unstable equilibria on $\mathcal{S}^{2}$ (see also Figure 1). It appears that almost global consensus holds for all the platonic graphs.

TABLE I. Number of failures to reach consensus on $\mathcal{S}^{2}$ over $10^{6}$ random trials for the five platonic graphs.

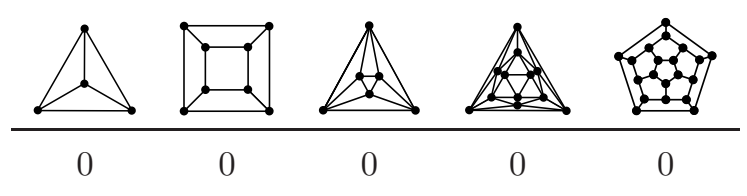

\section{REFERENCES}

[1] M. Mesbahi and M. Egerstedt, Graph Theoretic Methods in MultiAgent Networks. Princeton University Press, 2010.

[2] J. Markdahl, "Rigid-body attitude control and related topics," Ph.D. dissertation, KTH Royal Institute of Technology, 2015.

[3] W. Song, J. Markdahl, X. Hu, and Y. Hong, "Distributed control for intrinsic reduced attitude formation with ring inter-agent graph," in Proceedings of the 54th IEEE Conference on Decision and Control, 2015, pp. 5599-5604.

[4] D. A. Paley, "Stabilization of collective motion on a sphere," Automat$i c a$, vol. 45, no. 1, pp. 212-216, 2009.

[5] Y. Kuramoto, "Self-entrainment of a population of coupled non-linear oscillators," in International symposium on mathematical problems in theoretical physics, 1975, pp. 420-422.
[6] - Chemical oscillations, waves, and turbulence. Springer Science \& Business Media, 2012, vol. 19.

[7] N.A. Chaturvedi, A.K. Sanyal, and N.H. McClamroch, "Rigid-body attitude control: Using rotation matrices for continuous singularityfree control laws," IEEE Control Systems Magazine, vol. 31, no. 3, pp. $30-51,2011$

[8] R. Olfati-Saber, "Swarms on sphere: A programmable swarm with synchronous behaviors like oscillator networks," in Proceedings of the 45th IEEE Conference on Decision and Control. IEEE, 2006, pp. $5060-5066$.

[9] A. Sarlette, "Geometry and symmetries in coordination control," Ph.D. dissertation, Liège University, 2009.

[10] W. Li and M.W. Spong, "Unified cooperative control of multiple agents on a sphere for different spherical patterns," IEEE Transactions on Automatic Control, vol. 59, no. 5, pp. 1283-1289, 2014.

[11] R.W. Beard, J.R. Lawton, and F. Y. Hadaegh, "A coordination architecture for spacecraft formation control," IEEE Transactions on control systems technology, vol. 9, no. 6, pp. 777-790, 2001.

[12] J.R. Lawton and R.W. Beard, "Synchronized multiple spacecraft rotations," Automatica, vol. 38, no. 8, pp. 1359-1364, 2002.

[13] A. Rodriguez-Angeles and H. Nijmeijer, "Mutual synchronization of robots via estimated state feedback: a cooperative approach," IEEE Transactions on Control Systems Technology, vol. 12, no. 4, pp. 542554, 2004.

[14] A. Sarlette, R. Sepulchre, and N.E. Leonard, "Autonomous rigid body attitude synchronization," Automatica, vol. 45, no. 2, pp. 572-577, 2009.

[15] A. Sarlette, S. Bonnabel, and R. Sepulchre, "Coordinated motion design on Lie groups," IEEE Transactions on Automatic Control, vol. 55 , no. 5 , pp. $1047-1058,2010$.

[16] W. Ren, "Distributed cooperative attitude synchronization and tracking for multiple rigid bodies," IEEE Transactions on Control Systems Technology, vol. 18, no. 2, pp. 383-392, 2010.

[17] R. Tron, B. Afsari, and R. Vidal, "Riemannian consensus for manifolds with bounded curvature," IEEE Transactions on Automatic Control, vol. 58, no. 4, pp. 921-934, 2013.

[18] R. Hartley, J. Trumpf, Y. Dai, and H. Li, "Rotation averaging," International journal of computer vision, vol. 103, no. 3, pp. 267305, 2013.

[19] R. Tron and R. Vidal, "Distributed 3-D localization of camera sensor networks from 2-D image measurements," IEEE Transactions on Automatic Control, vol. 59, no. 12, pp. 3325-3340, 2014.

[20] J. Thunberg, W. Song, E. Montijano, Y. Hong, and X. Hu, "Distributed attitude synchronization control of multi-agent systems with switching topologies," Automatica, vol. 50, no. 3, pp. 832-840, 2014.

[21] N. Matni and M.B. Horowitz, "A convex approach to consensus on $\mathrm{SO}(\mathrm{n})$," in Proceedings of the 52nd Annual Allerton Conference on Communication, Control, and Computing, 2014, pp. 959-966.

[22] R. Tron, B. Afsari, and R. Vidal, "Intrinsic consensus on $\mathrm{SO}(3)$ with almost-global convergence," in Proceedings of the 51th IEEE Conference on Decision and Control, 2012, pp. 2052-2058.

[23] J. Markdahl, X. Hu, and W. Song, "Global and invariant aspects of consensus on the $n$-sphere," in The 22nd International Symposium on Mathematical Theory of Networks and Systems, 2016, submitted.

[24] D. Hinrichsen and A.J. Pritchard, Mathematical Systems Theory I: Modelling, State Space Analysis, Stability and Robustness. Springer, 2005.

[25] Y.A. Kuznetsov, Elements of applied bifurcation theory. Springer, 2013.

[26] M. Shub, Global stability of dynamical systems. Springer, 2013.

[27] S.P. Bhat and D.S. Bernstein, "A topological obstruction to continuous global stabilization of rotational motion and the unwinding phenomenon," Systems \& Control Letters, vol. 39, no. 1, pp. 63-70, 2000.

[28] S.S. Sastry, Nonlinear systems: analysis, stability, and control. Springer, 1999.

[29] R.A. Horn and C.R. Johnson, Matrix analysis. Cambridge University Press, 2012.

[30] H.K. Khalil, Nonlinear systems. Prentice Hall, 2002.

[31] M.E. Muller, "A note on a method for generating points uniformly on n-dimensional spheres," Communications of the ACM, vol. 2, no. 4, pp. 19-20, 1959. 